\title{
SIMULAÇÃO COM O SOFTWARE ARENA PARA O USO DE UM LEITOR DE CÓDIGO DE BARRAS NO PROCESSO DE DIGITAÇÃO DE BOLETOS BANCÁRIOS PARA PAGAMENTOS EM UMA CENTRAL DE SERVIÇOS COMPARTILHADOS
}

\author{
Ana Carolina da Costa Rodrigues (FIC/UNIS) - costa.anacarolinar@ gmail.com \\ Lucas Rodrigues Lobo (FIC/UNIS) - lucasrolobo@ hotmail.com \\ Lara Rodrigues Ferraz (FIC/UNIS) - lara_rodrigues@ hotmail.com \\ Caio Ravaglia Vicente (FIC/UNIS) - c15.ravaglia@gmail.com \\ Luis Antonio Paixão de Rezende (FIC/UNIS) - luis.rezende@ unis.edu.br
}

RESUMO: O estudo possui como objetivo, aplicar através de simulação computacional o uso do leitor de código de barras no processo de digitação de boletos bancários de uma área pertencente a um Centro de Serviços Compartilhados de uma empresa do setor elétrico localizado na cidade de Cataguases, Minas Gerais, mapeando as atividades pertinentes do atual processo e realizando uma simulação no software Arena para redução do retrabalho e maior eficiência na digitação, a fim de analisar o resultado da simulação para auxiliar na tomada de decisão quanto ao uso. Com a utilização do leitor de código de barras observou-se um ganho de $24 \%$ na eficiência do processo bem como a eliminação do retrabalho.

Palavras-chave: Simulação; Software Arena; Processo; Boletos Bancários; Decisão.

\section{INTRODUÇÃO}

Através da evolução tecnológica, em considerável velocidade e a competitividade atingindo um patamar global, é necessário buscar constantemente a otimização de processos. Redução de custos, melhoramento da qualidade, inovação e otimização de tomadas de decisões são aspectos essenciais para que as empresas se mantenham firmes e ganhem mercado. Existem medidas, nas quais as empresas podem tomar para terem resultados competitivos e sobressaírem em relação aos seus concorrentes, consequentemente aumentando os lucros.

A busca pelo aprimoramento de seus processos é uma delas, contudo para que tais medidas sejam adotadas, é preciso ter o conhecimento de como elas irão afetar a empresa e se o investimento não acarretará em um futuro prejuízo.

Investimentos em processos e tecnologias acarretam em custos além de serem arriscados, pois são itens com incertezas, a utilização sistemas de informação para automatizar seus processos de trabalho, para armazenar e recuperar dados e para compartilhar informações (MORESI; RAMOS; PRADO, 2010). Porém, a utilização de um processo de simulação pode subsidiar a eficiência dos investimentos, além de diminuir as dúvidas existentes e identificar possíveis riscos. Deste modo, PRADO (2010) definiu a simulação como uma técnica de solução 
de um problema pela análise de um modelo que descreve o comportamento do sistema.

O estudo tem como objetivo aplicar a simulação computacional no processo de solicitação de pagamento, mediante a utilização de leitores de código de barras em uma área específica pertencente a uma empresa do setor elétrico na cidade de Cataguases. Para atender o objetivo do estudo foi necessário descrever quais as atividades relacionadas ao processo produtivo do setor da empresa, desenvolver a simulação das atividades no software Arena com o intuito de estabelecer uma comparação entre o cenário atual e o futuro, como forma de subsidiar uma eventual mudança.

\section{DESENVOLVIMENTO 2.1 Metodologia}

Como objeto de estudo do trabalho, foi selecionado o setor de Infraestrutura que pertence a um Centro de Serviços Compartilhados da respectiva empresa. Sendo assim, de acordo com Marconi e Lakatos (2013), as características básicas da pesquisa são de exploração técnica, sistemática e exata, pois utiliza conhecimentos teóricos já existentes, tendo a formulação do problema e de hipóteses planejadas cuidadosamente, registrando sistematicamente os dados e os analisando com a maior exatidão possível.

Para o desenvolvimento da pesquisa, foi necessário à obtenção de uma amostragem, que corresponde a um "subgrupo da população, do qual são coletados os dados que deve ser representativo dessa população", (SAMPIERI; COLLADO; LUCIO, 2013, p. 192). Esta amostragem foi relacionada aos tempos dos processos envolvidos no setor estudado. Para isso, ocorreu o mapeamento de todo os processos, bem como uma a coleta de informações relacionadas aos tempos de cada uma das operações referentes à digitação dos boletos no sistema, onde foi cronometrado cada atividade individualmente, obtendo o tempo que cada colaborador levou para efetuar sua função.

No que diz respeito ao software de simulação, foi utilizada a versão acadêmica do software Arena, um programa computacional de simulação gratuito da empresa Rockwell Softwares, uma empresa que é sediada em Milwaukee (EUA) e é uma das principais fornecedoras de soluções em automação do mundo. A versão utilizada apresenta as funcionalidades necessárias para o decorrer da pesquisa. 


\subsection{Empresa Objeto de Estudo}

A área abordada no estudo é composta por três frentes de trabalhos: Facilities, Frota e Viagens totalizando cerca de 304 colaboradores. A mesma possui uma célula de trabalho chamado de núcleo de processamento de notas, a qual que é composta de 12 colaboradores, cuja responsabilidade é de fazer a digitação de aproximadamente 4.500 documentos por mês, como notas fiscais de serviços e venda de materiais, alugueis, IPVA, contas de telefone entre outros. Parte desses documentos são pagos através de boletos bancários que são cerca de 705 boletos mensais.

Através do mapeamento de processo, foi identificado que a célula é dividida em 3 frentes, Back Office (4), Digitação (5) e Abertura de chamados (3).

A equipe de Back office tem as seguintes atribuições:

Fazer a triagem dos documentos e analise;

Acompanhamento de indicadores;

Liberação de documentos para aprovação dos gestores;

Digitação

Digitação dos documentos correspondentes;

Abrir chamados de pagamento para o setor financeiro.

\subsection{Mapeamentos de Processos}

Os processos podem ser compostos por uma série de atividades, que são desenvolvidas pelas pessoas dentro de uma organização. Nesse sentido, os processos e atividades só devem existir se agregam valor a atividade da organização, uma vez que necessitam, as atividades, assim como os processos, estarem interligadas entre si. Em consonância com o exposto, a maioria dos processos pode ser aperfeiçoada, se alguém pensar em um modo de fazê-lo e implementá-lo de maneira eficaz (KRAJEWSKI; RITZMAN; MALHOTRA, 2009).

Por se tratar de um componente essencial para o gerenciamento e comunicação, o mapeamento de processos pode ainda permitir a redução de custos na prestação de serviços, a redução nas falhas de integração entre sistemas e melhora do desempenho da organização. Além 
de ser uma excelente ferramenta para possibilitar o melhor entendimento dos processos atuais e eliminar ou simplificar aqueles que necessitam de mudanças (GOMES, et al., 2015).

O mapeamento de processos vem ao encontro da identificação dos principais passos e decisões em um fluxo de trabalho de rotina de forma visual. Também controla o fluxo de informações, materiais e documentos envolvidos no processo e esclarece tarefas, decisões e ações que são necessárias em determinados pontos no tempo. Além disso, mapas de processos representam os papéis de uma variedade de partes interessadas que impacto ou atuar no processo (BARBROW; HARTLINE, 2015).

\subsection{Simulação}

Para Correa, Mello e Pereira (2014) o método de simulação consiste em uma forma de alocar tarefas aos operadores, distribuindo as atividades entre trabalhadores que estão sobrecarregados com os mais ociosos, a fim de encontrar um equilíbrio nos tempos das atividades. Chwif (2012) explica que um modelo de simulação é uma abstração da realidade, que se aproxima do verdadeiro comportamento do sistema, mas sempre mais simples que o sistema real. Ao se efetuar certos tipos de estudo de planejamento, alguns problemas relacionados a dimensionamento ou fluxo cuja solução aparentemente é complexa, podem aparecer (PRADO, 2009).

Assim, o objetivo maior que leva as pessoas a optarem pelo uso da simulação, é o fato de que ao se implantar algo novo se gera um custo, e sem um bom planejamento, é possível que tal implementação acabe gerando um prejuízo para a organização.

\section{RESULTADOS E DISCUSSÕES}

Foi identificado que as atividades necessárias para digitação de um boleto bancário são: Preenchimento de Cabeçalho; Preenchimento de Dados do Boleto; Preenchimento do Código de Barras; Campo de Inserir Anexos e Campo para Inserir Área Conta. A fim de garantir a análise e verificar o tempo médio total do processo, foi medido o tempo para cada atividade em um período de 9 dias conforme mostra no Quadro 1: 
Quadro 1 - Cronometragem dos tempos para execução de cada etapa do processo de pagamento de boleto

\begin{tabular}{|c|c|c|c|c|c|c|c|c|c|}
\hline Processo & $1^{\circ}$ dia & $\overline{2^{\circ} \text { dia }}$ & $3^{\circ}$ dia & $4^{\circ} \mathrm{dia}$ & $5^{\circ}$ dia & $6^{\circ}$ dia & $7^{\circ} \mathrm{dia}$ & $8^{\circ} \mathrm{dia}$ & $9^{\circ}$ dia \\
\hline Preenchimento de Cabeçalho & 16 & 18 & 17 & 17 & 19 & 16 & 17 & 17 & 19 \\
\hline Preenchimento de Dados do Boleto & 42 & 45 & 41 & 44 & 42 & 40 & 41 & 42 & 42 \\
\hline Preenchimento do Código de Barras & 21 & 26 & 27 & 23 & 22 & 21 & 20 & 22 & 26 \\
\hline Campo de Inserir Anexos & 17 & 14 & 12 & 11 & 23 & 26 & 22 & 19 & 20 \\
\hline Campo para Inserir Área Conta & 16 & 14 & 15 & 17 & 18 & 14 & 14 & 21 & 14 \\
\hline Média dos Tempos (em segundos) & 112 & 117 & 112 & 112 & 124 & 117 & 114 & 121 & 121 \\
\hline Tempo Total (em segundos) & 116,67 & & & & & & & & \\
\hline
\end{tabular}

Fonte: Elaborado pelo autor (2018)

Para a simulação no software foi construído um cenário (figura 1) que a cada 900 segundos à entrada de um boleto para digitação, levando em consideração 22 dias de trabalho e 8 horas diárias de carga de trabalho. Pelo fato da digitação ser manual é comum o erro, tão logo esse erro pode proporcionar o reprocesso de digitação ou perda do documento devido à data de vencimento.

Figura 1 - Desenho do processo de pagamento de boletos no software Arena

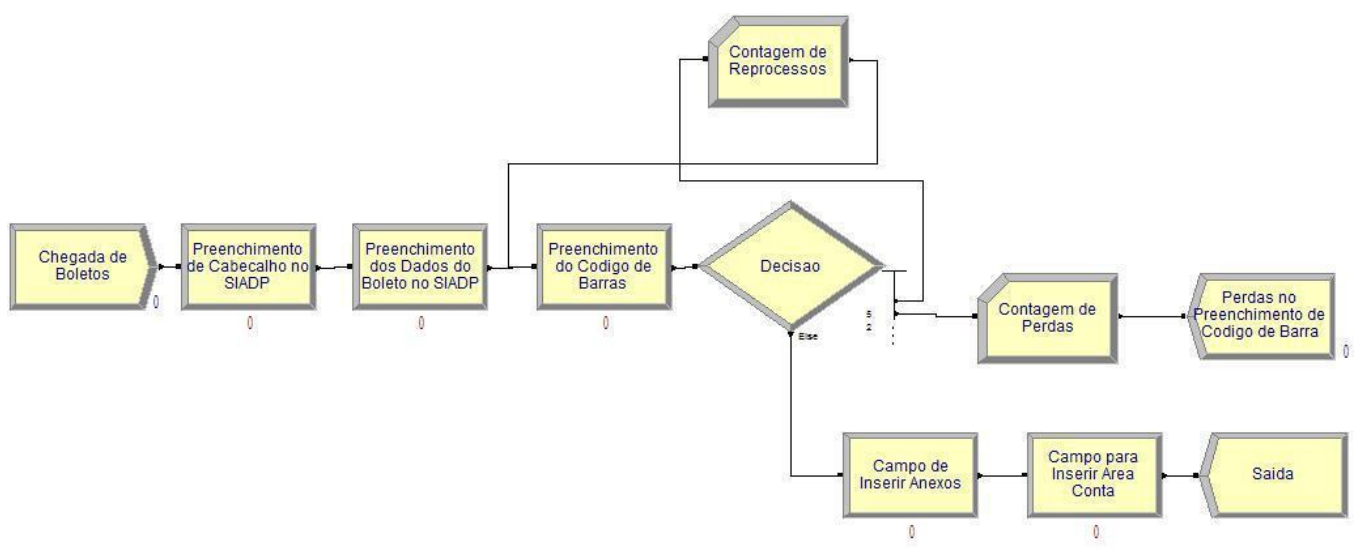

A média mensal de digitação é de 705 boletos e cerca 7\% do total apresentam retrabalho e perda devido ao erro de digitação. Portanto para o problema foi considerado de $5 \%$ de retrabalho e $2 \%$ de perda, logo o retorno do Arena foi de 40 boletos com retrabalho e 15 boletos com perda, totalizando 55 erros. 
Um novo cenário nas mesmas condições foi proposto, porém com a utilização do leitor do código de barras para substituir o processo de digitação manual e com o intuito da eliminação do retrabalho. Foi observado que o tempo da leitura no sistema é de aproximadamente 2 segundos, com essa alteração, novos tempos foram estimados conforme mostra o Quadro 2:

Quadro 2 - Cronometragem dos tempos para execução de cada etapa do processo de pagamento de boletos com a utilização do leitor de código de barras

\begin{tabular}{|l|c|c|c|c|c|c|c|c|c|}
\hline \multicolumn{1}{|c|}{ Processo } & $\mathbf{1}^{\circ} \mathbf{d i a}$ & $\mathbf{2}^{\circ} \mathbf{d i a}$ & $\mathbf{3}^{\circ} \mathbf{d i a}$ & $\mathbf{4}^{\circ} \mathbf{d i a}$ & $\mathbf{5}^{\circ} \mathbf{d i a}$ & $\mathbf{6}^{\circ} \mathbf{d i a}$ & $\mathbf{7}^{\circ} \mathbf{d i a}$ & $\mathbf{8}^{\circ} \mathbf{d i a}$ & $\mathbf{9}^{\circ} \mathbf{d i a}$ \\
\hline Leitor do Código de Barras & 2 & 2 & 2 & 3 & 3 & 2 & 1 & 3 & 3 \\
\hline Campo de Inserir Anexos & 17 & 14 & 12 & 11 & 23 & 26 & 22 & 19 & 20 \\
\hline Campo para Inserir Área Conta & 16 & 14 & 15 & 17 & 18 & 14 & 14 & 21 & 14 \\
\hline Média dos Tempos (em segundos) & 93 & 93 & 87 & 92 & 105 & 98 & 95 & 102 & 98 \\
\hline Média Total (em segundos) & 95,889 & &
\end{tabular}

Fonte: Elaborado pelo autor (2018)

Com os novos tempos medidos em cada etapa do processo de pagamento de boletos, utilizando o leitor de código de barras, foi simulado um novo desenho no software Arena, conforme mostra a Figura 2:

Figura 2- Desenho do processo de pagamento de boletos com a utilização do leitor de código de barras no software Arena

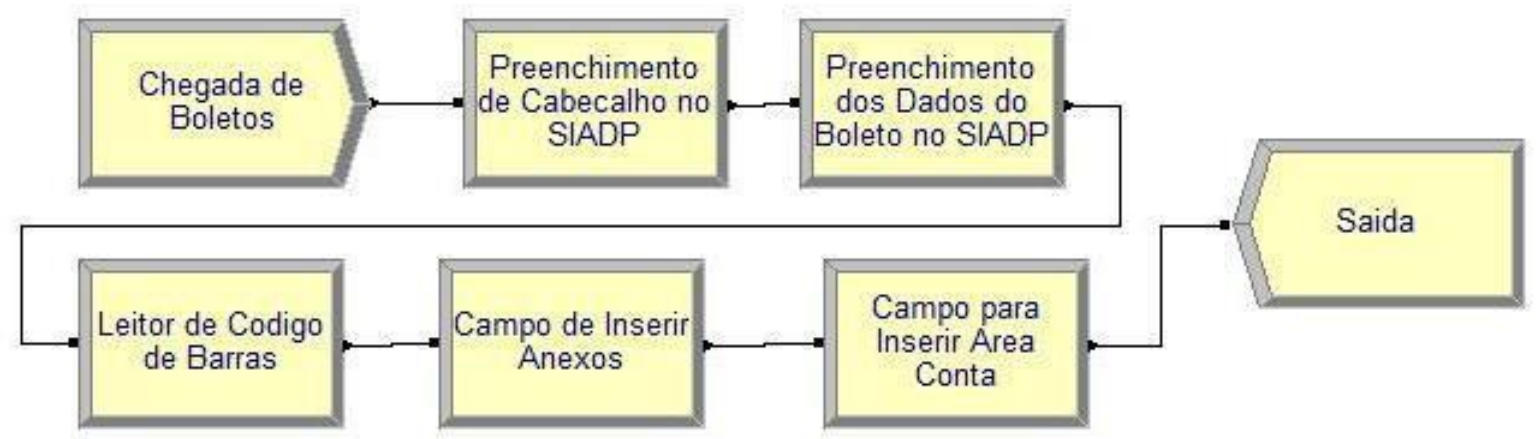

Com a eliminação do retrabalho, o Arena não retornou o número estimado de boletos com erros. O novo cenário simulado no software demonstrou que são atendidos 200 chamados a mais pelos digitadores com a utilização do leitor de código de barras e melhoria no tempo 
estimado do processo, conforme visto na figura 3.

Figura 3 - Número de boletos digitados para pagamento com a aplicação do leitor de código de barras.

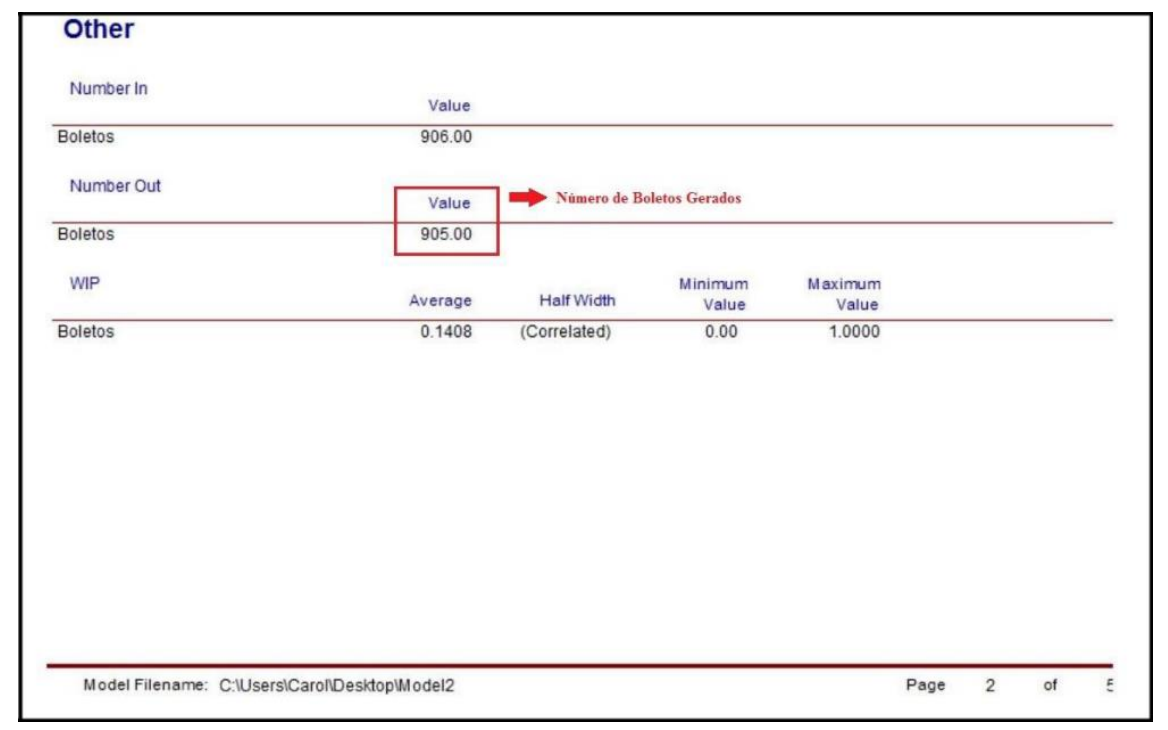

Dessa forma foi elaborado um quadro comparativo para os dois cenários levando em consideração o tempo total do processo. Conforme o Quadro 3, foi cronometrado os tempos para digitação sem a utilização do leitor de código de barras:

Quadro 3 - Tempos totais do Cenário 1, sem a utilização do leitor de código de barras.

\begin{tabular}{|l|r|}
\hline Total de Notas Mês & 705 \\
\hline Tempo Médio Total & 22,8472 \\
\hline Total de Notas Redigitação & 55 \\
\hline Tempo Total Gasto com Redigitação & 1,78241 \\
\hline Tempo Total Gasto no Processo (horas) & 24,6296 \\
\hline Jornada de Trabalho (dias) & 3,0787 \\
\hline
\end{tabular}

Fonte: Elaborado pelo autor (2018)

No Quadro 4, foi cronometrado os tempos gastos na digitação dos boletos utilizando o leitor de código de barras no processo: 
Quadro 4 - Tempos totais do Cenário 2, com a utilização do leitor de código de barras.

\begin{tabular}{|l|r|}
\hline Total de Notas Mês & 705 \\
\hline Tempo Médio Total & 18,7782 \\
\hline Total de Notas Redigitação & 0 \\
\hline Tempo Total Gasto com Redigitação & 0 \\
\hline Tempo Total Gasto Processo (horas) & 18,7782 \\
\hline Jornada de Trabalho (dias) & 2,34728 \\
\hline
\end{tabular}

Fonte: Elaborado pelo autor (2018)

Com a utilização do leitor de código de barras foi notado um ganho de $24 \%$ na eficiência do processo bem como a eliminação do retrabalho. Foram considerados os custos necessários para implantação do leitor de código de barras no processo e adaptação em sistema.

\section{CONCLUSÃO}

O objetivo do estudo foi abordar a simulação no software Arena para verificar o fluxo da digitação de boletos para pagamento do setor da Infraestrutura do Centro de Serviços Compartilhados e elaborar uma proposta de melhoria no mesmo, com o objetivo de tornar mais eficiente à produção e reduzir o retrabalho utilizando um leitor de código de barra.

O modelo de simulação desenvolvido respondeu aos objetivos estabelecidos, gerando um ganho de $24 \%$ na eficiência do processo. Este modelo também pode ser utilizado como em outras empresas, tendo como base o referencial teórico, o estudo abordado sendo que através dos objetivos propostos foi possível elaborar cenário aproveitando melhor os recursos e eficiência do setor atrás da força de trabalho destinado a nova operação.

Foi obtido com a proposta de modelo, o resultado para uma possível mudança no fluxo da digitação, onde se verificou a possível diminuição do retrabalho e aumento da eficiência na digitação de boletos bancários. Deste modo, a simulação satisfez as expectativas, sendo considerada uma boa técnica para testar o novo fluxo do setor, tendo como vantagem visualizar 
o comportamento das atividades relacionadas ao setor com um baixo custo envolvido, opção no qual o software proporciona. Como estudo futuro, é sugerida a realização de uma análise semelhante nas demais etapas do processo.

\section{REFERÊNCIAS BIBLIOGRÁFICAS}

BORSHCHEV, A.; FILIPPOV, A. From system dynamics and discrete event to practical agent based modeling: Reasons, techniques, tools. In: International Conference Of The System Dynamics Society. Jul 25-29, Oxford, England: Keble College, 2004.

CAIXETA-FILHO, J. V. Pesquisa operacional: técnicas de otimização aplicadas a sistemas agroindustriais. 2. ed. São Paulo: Atlas, 2004.

CHASE, R.B.; AQUILANO, N.J.; JACOBS, F.R. Administração da produção e operações para vantagens competitivas. $11^{a}$ Ed. São Paulo: McGraw-Hill interamericana do Brasil, 2006.

CORREA, J. E.; MELLO, C. H.; PEREIRA, T. F. Uso de simulação de eventos discretos para avaliação de uma linha de montagem de uma empresa do ramo automotivo e os impactos do fator humano. In: Encontro Nacional de Engenharia de Produção, 34, 2014, Curitiba, Anais...Curitiba: ENEGEP, 2014.

D’ASCENÇÃO, L.C.M. Organização, Sistemas e Métodos: análise, redesenho e informatização de processos administrativos. São Paulo: Atlas, 2001.

GUIMARAES, I F.; PRAZERES, I. P. Utilização da simulação computacional como ferramenta para gestão de pronto atendimentos hospitalares. In: Encontro Nacional de Engenharia de Produção, 34, 2014, Curitiba, Anais... Curitiba: ENEGEP, 2014.

HARREL, C. R.; MOTT, J. R. A.; BATEMAN, R. E.; BOWDEN, R. G.; GOGG T. J. Simulação: otimizando os sistemas. 2. ed. São Paulo: IMAM, 2002.

KIPPER, L.M.; ELLWANGER, M. C.; JACOBS, G.; NARA, E. O. B.; FROZZAS, R. 2011. Gestão por processos: Comparação e análise entre metodologias para implantação da gestão orientada a processos e seus principais conceitos. Revista Tecno-Lógica, v. 15, n. 2, p. 89-99, 2011.

LOESCH, C.; HEIN, N. Pesquisa operacional: fundamentos e modelos. 1. ed. São Paulo: Saraiva, 2009. MARCONI, M. A.; LAKATOS, E. M. Técnicas de pesquisa: planejamento e execução de pesquisa, amostragem e técnicas de pesquisa, elaboração, analise e interpretação de dados. 7. ed. São Paulo: Atlas, 2013.

MOREIRA, D. A. Administração da produção e operações. 1. ed. São Paulo: Pioneura Thomson Learning, 2004.

MORESI, E.A.D.; RAMOS, R.G.C.; PRADO, H.A.; Organizational information mapping: a study at Embrapa. TransInformação, v. 22, n. 2, p.110-110, 2010.

PRADO, D. Usando o Arena em Simulação. Editora DG, Belo Horizonte.

SAMPIERI, R. H.; COLLADO, C. F.; LUCIO, M. D. Metodologia de pesquisa. 5. ed. Porto Alegre: 